\title{
High Order Corrections to the Time-Independent Born-Oppenheimer Approximation II: Diatomic Coulomb Systems
}

\author{
George A. Hagedorn* \\ Department of Mathematics and Center for Transport Theory and Mathematical Physics. Virginia \\ Polytechnic Institute and State University, Blacksburg, Virginia 24061-4097, USA
}

\begin{abstract}
We study the bound states of diatomic molecular systems. We prove that if the nuclear masses are proportional to $\varepsilon^{-4}$ then certain eigenvalues and eigenvectors of the Hamiltonian have asymptotic expansions to arbitrarily high order in powers of $\varepsilon$, as $\varepsilon \rightarrow 0$. The zeroth through fourth order terms in the expansions for the eigenvalues are those of the well-known Born-Oppenheimer approximation. The fifth order term is zero.
\end{abstract}

\section{Introduction}

In this paper we study the quantum mechanics of diatomic molecules and ions by exploiting the fact that the nuclear masses are much larger than the electronic masses. We prove that if the nuclear masses are proportional to $\varepsilon^{-4}$, then certain bound state energies and wave functions have asymptotic expansions to arbitrarily high orders in powers of $\varepsilon$ as $\varepsilon \rightarrow 0$. In an earlier paper [15], we proved this for molecular type systems with smooth potentials. The present paper is the extension of [15] to overcome the difficulties associated with Coulomb potentials.

Although we will discuss only Coulomb systems, our techniques clearly extend to diatomic molecular and ionic type systems with spherically symmetric dilation analytic potentials [20] that are relatively operator bounded with respect to the Laplacian [20]. We have concentrated on Coulomb systems for concreteness and because they are physically the most interesting.

The principal difficulty in extending the results of [15] to Coulomb systems is that the Coulomb singularities can give rise to cusps in the electronic wave functions at the positions of the nuclei. The techniques of [15], require certain derivatives of the electron wave functions that do not exist in the Coulomb case. We overcome this difficulty by noticing that the required derivatives are directional derivatives, and that by altering the expansion we can avoid the "bad" directions where the

* Supported in part by the National Science Foundation under grant number DMS-8601536 
derivatives do not exist. In the appropriate "good" directions the electron wave functions are analytic.

In order to make the discussion as concrete as possible, we will make changes of independent variables so that the "good" directions are some of the new coordinate directions. That way, to lowest order, the Born-Oppenheimer expansion will look like a separation of variables.

Approximations of the type we are studying were first developed in 1927 by Born and Oppenheimer [4]. They formally showed that molecular energy levels had asymptotic expansions through the fourth order in $\varepsilon$, and that the non-zero terms in the expansions had direct physical interpretations. They stopped at fourth order for various physical reasons, and seem to have been unaware that some derivatives they would have needed at fifth order did not exist. The physical basis for their approximations can be summarized as follows: The small mass electrons move very rapidly compared to the large mass nuclei. As a result, the adiabatic approximation fairly accurately describes the electron motion, i.e., on a short time scale, the electrons hardly notice the motion of the nuclei, and on a large time scale, they rapidly adjust their motion in response to the changing positions of the nuclei. In addition, the nuclear motion is approximately semiclassical due to the large nuclear masses.

The disparity between the periods for the electronic and nuclear motions leads to a separation in the energies of these motions. Roughly speaking, as $\varepsilon$ tends to zero, the energy terms decompose as follows: The electronic energy is $O(1)$; the molecular vibrational energy is $O\left(\varepsilon^{2}\right)$; and the molecular rotational energy is $O\left(\varepsilon^{4}\right)$. There are additional $O\left(\varepsilon^{4}\right)$ terms (anharmonic corrections to the nuclear vibrational energies, and the lowest order term involving the coupling of electronic and nuclear motions). As we shall see below, the terms of orders $\varepsilon^{1}, \varepsilon^{3}$, and $\varepsilon^{5}$ all vanish. The terms of order $\varepsilon^{6}$ and higher involve complicated interactions between the electronic and nuclear motions, and they do not have simple physical interpretations. Although we have not computed the $\varepsilon^{7}$ terms in any specific examples, we believe that they are generically non-zero. In particular, we see no reason for all the odd order terms to vanish.

From the above discussion and the discussions in most physical textbooks on the subject, one would be led to believe that the disparity between time scales is the basis for the validity of the Born-Oppenheimer approximation. This is not the proper intuition, even in the time dependent approximation [14]. The crucial ingredient is a disparity in spatial scales. In the time that it takes the electrons to move a unit distance, the nuclei move a distance of order $\varepsilon$. As a result, the appropriate technique for analyzing the motion is the "method of multiple scales" applied to the appropriate spatial variables. Born and Oppenheimer [4] were clearly aware of the role of spatial scaling, but did not have a clean formalism for dealing with more than one scale in the same variable.

There has been very little rigorous mathematical work concerning the validity of the Born-Oppenheimer approximation until recently. In the last decade, Seiler [21] has worked out a simple exactly soluble Born-Oppenheimer type model involving coupled harmonic oscillators, and Aventini, Combes, Duclos, Grossman, and Seiler [5-7] have rigorously proved the fourth order results of [4]. The only 
other related mathematical work on this subject (of which we are aware) is the author's own work $[13-15]$. In $[13,14]$ the time-dependent Born-Oppenheimer expansion is shown to be asymptotic to all orders in $\varepsilon$ to the exact solution of the Schrödinger equation if the potentials are smooth. The paper [15] proves the analogous time-independent result.

The papers $[6,7]$ of Combes, Duclos, and Seiler involve some very clever techniques for analyzing the discrete spectrum of a molecular Hamiltonian. By using a Feshbach projection technique, they have computed rigorous upper and lower bounds for the eigenvalues. As $\varepsilon \rightarrow 0$, the upper and lower bounds agree through fourth order, and the asymptotics of the lowest finitely many eigenvalues can be computed. Unfortunately, the estimates are not uniform, in the sense that as one looks at higher eigenvalues, the estimates become poorer. Thus, only the bottom of the spectrum is completely described. The estimates do guarantee the presence of eigenvalues near certain higher energies, but do not preclude the possibility that eigenvalues with other asymptotics might be present above the first finitely many eigenvalues.

We regard the papers $[6,7]$ as being very deep, careful analyses of the low-lying spectrum in a very singular perturbation problem. In addition, the techniques are capable of handling the technical problems associated with Coulomb potentials through fourth order. The crucial arguments of $[6,7]$ employ some clever non-linear techniques to establish the lower bounds.

In contrast, we will use linear methods to produce high order "quasimodes." That is, we will produce solutions to the inequality

$$
\|H(\varepsilon) \Psi(\varepsilon)-\mathscr{E}(\varepsilon) \Psi(\varepsilon)\| \leqq C_{N} \varepsilon^{N},
$$

where $N$ is arbitrarily large and $C_{N}$ is appropriately chosen. The existence of such quasimodes guarantees that either $\mathscr{E}(\varepsilon)$ is in the spectrum of the self-adjoint operator $H(\varepsilon)$, or the norm of the resolvent is least $\varepsilon^{-N} / C_{N}$. Thus, $H(\varepsilon)$ must have some spectrum in the interval $\left[\mathscr{E}(\varepsilon)-C_{N} \varepsilon^{N}, \mathscr{E}(\varepsilon)+C_{N} \varepsilon^{N}\right]$. This proves the presence of spectrum near certain energies, but does not preclude the possibility that there is also other spectrum present. In addition, we have the same uniformity problem as $[6,7]$. However, by combining our results with other results, more detailed information can be obtained. For example:

1. By combining our results with the HVZ Theorem [20], we can be sure that certain quasimodes correspond to discrete eigenvalues: The HVZ Theorem characterizes the bottom $\Sigma$ of the essential spectrum. Quasimode estimates that guarantee spectrum below $\Sigma$, guarantee the presence of discrete eigenvalues.

2. By combining our results with those of $[6,7]$, it is easy to see that our high order quasimodes completely describe the asymptotics of the lowest finitely many eigenvalues to arbitrarily high order.

In the next section, we will precisely state our results. In Sect. 3, we will give a formal computation of the quasimodes by using the method of multiple scales. In the fourth section we will rigorously justify all the steps of the formal computation.

Remarks. 1. In contrast to the approach of [15], we will separate the total angular 
momentum dependence before beginning the expansion in powers of $\varepsilon$. The separation will be convenient in the rotating, dilating coordinate system we will use because the total angular momentum operator $J^{2}$ and the $z$-component $J_{z}$ will be given by simple expressions in the new coordinates. By using the rotating coordinates without the dilations, one can redo the analysis of [15] in what is perhaps a more natural way. This leads to more complicated formulas, but one can avoid the infinite degeneracies that are only broken at fourth order in [15].

2. Born and Oppenheimer only considered nondegenerate electronic states. We can improve upon this by allowing the degeneracy that gives rise to the phenomenon of " $\Lambda$-doubling." To low orders in our expansion, the component of the electron angular momentum about the internuclear axis, $L_{z^{\prime}}$, is conserved. There are two electronic states with the same energy corresponding to $L_{z^{\prime}}= \pm \Lambda$ if $\Lambda \neq 0$. The phenomenon of $\Lambda$-doubling is the splitting of these energy levels. Generically, the splitting occurs at order $\varepsilon^{8 ! \Lambda !}$.

3. We have split Sect. 3 into two parts. In the first part we will explicitly write out the case of one electron. In the second part we will show that the formal expansion for two or more electrons can be developed, without explicitly presenting all the formulas. The addition of more electrons makes the formulas more complicated, but no further new ideas are required.

4. We are not particularly fond of our proof! We believe there ought to be a proof similar to the one used in [22] to study semiclassical asymptotics. When we tried to mimic the lower bound estimates of [22], we found ourselves facing the difficulties that caused the authors of $[6,7]$ to resort to nonlinear techniques.

5. The ideas that we use in this paper can also be used to extend the time dependent results of [14] to diatomic Coulomb systems in two ways. First, one could diagonalize the total angular momentum and use the complex coherent states of [14] in the variable $R$. This is the most straightforward way to extend $[14]$ by using the ideas of the present paper. This is not the physically most natural result, but it might give some phase shift information. The second extension would use the complex coherent states in the variables $R, \theta$, and $\varphi$. We plan to publish results of this type for polyatomic systems by using the ideas of [16], rather than the ideas of the present paper.

6. The present paper's ideas do not extend easily to polyatomic systems. One could try to use the ideas of [16], but it is not clear how to implement those ideas globally, which must be done in the time independent situation.

7. In some molecules, spin effects are very important. In fact, in terms of the energy, they can dominate the rotational effects. For Coulomb systems we do not know how to include these terms because of self-adjointness problems. Even in the case of the Hydrogen atom, we do not know what the physically correct Hamiltonian is if spin-orbit effects are included. The formal operator one can find in the physics textbooks is not essentially self adjoint. The difficulty comes from the singularity in the term proportional to $L \cdot S / r^{3}$. See [23].

8. In our approach, one can impose Fermi statistics on the electrons. Remark 7 describes the only obstacle that prevents us from dealing with a completely physically realistic model of molecules. Only for convenience have we ignored the Fermi statistics for the electrons. However, if the Fermi statistics are imposed, the 
lowest electronic state of the system might have $\Lambda \neq 0$. In that case, some of the remarks of Sect. 2 must be modified.

\section{Notation and Results}

In this section we present precise statements of our main theorems and develop notation.

We consider an $N$-body quantum mechanical system of particles whose masses are $M_{1} \varepsilon^{-4}, M_{2} \varepsilon^{-4}$, and $m_{j}(j=3,4, \ldots, N)$. We assume that particles 1 and 2 interact via a non-zero repulsive Coulomb potential, and that the all the other potentials in the problem are also Coulombic. We refer to such a system a diatomic molecule. Particles 1 and 2 are called the nuclei; particles $3,4, \ldots, N$ are called the electrons. The Hamiltonian for a diatomic molecule is

$$
\tilde{H}(\varepsilon)=-\frac{\varepsilon^{4}}{2 M_{1}} \Delta_{1}-\frac{\varepsilon^{4}}{2 M_{2}} \Delta_{2}-\sum_{j=3}^{N} \frac{1}{2 m_{j}} \Delta_{j}+\sum_{i<j} \frac{z_{i j}}{\left|x_{i}-x_{j}\right|}
$$

on $L^{2}\left(\mathbb{R}^{3 N}\right)$, with $z_{12}>0$. We choose a clustered Jacobi coordinate system [20] in which the first three coordinates are the vector $X$, from the first nucleus to the second, and the second three coordinates are the vector $\zeta_{1}$, from the center of mass of the nuclei to the center of mass of the electrons. Then we remove the center of mass dependence [20] from $\tilde{H}(\varepsilon)$ to obtain

$$
H(\varepsilon)=-\frac{\varepsilon^{4}}{2 M} \Delta_{X}-\sum_{j=1}^{N-2} \frac{1}{2 v_{j}(\varepsilon)} \Delta_{\zeta_{j}}+V\left(X, \zeta_{1}, \zeta_{2}, \ldots, \zeta_{N-2}\right)
$$

on $L^{2}\left(\mathbb{R}^{3 N-3}\right)$. The reduced masses $v_{j}(\varepsilon)$ are analytic in $\varepsilon^{4}$ and approach non-zero values $\mu_{j}$ as $\varepsilon$ tends to zero. For convenience, we assume that $M=1$, and we define $r$ to be the vector $\left(\zeta_{1}, \zeta_{2}, \ldots, \zeta_{N-2}\right) \in \mathbb{R}^{3 N-6}$. We define the electron Hamiltonian $h(X)$ to be the operator valued function

$$
h(X)=-\sum_{j=1}^{N-2} \frac{1}{2 \mu_{j}} \Delta_{\zeta_{3}}+V\left(X, \zeta_{1}, \zeta_{2}, \ldots, \zeta_{N-2}\right)
$$

on $L^{2}\left(\mathbb{R}^{3 N-6}, d r\right)$. By using the direct integral decomposition $L^{2}\left(\mathbb{R}^{3 N-3} d X d r\right)=$ $\int_{\mathbb{R}^{3}} \oplus L^{2}\left(\mathbb{R}^{3 N-6}\right) d X$, we define $h$ to be the operator on $L^{2}\left(\mathbb{R}^{3 N-3} d X d r\right)$ which is the direct integral of the fiber operators $h(X)$. In addition, we define an operator $D(\varepsilon)$ by the relation

$$
\varepsilon^{4} D(\varepsilon)=-\sum_{j=1}^{N-2}\left(\frac{1}{2 v_{j}(\varepsilon)}-\frac{1}{2 \mu_{j}}\right) \Delta_{\zeta j} .
$$

Note that $D(\varepsilon)$ is a second order differential operator whose coefficients are analytic in $\varepsilon^{4}$. With this notation, we have

$$
H(\varepsilon)=-\frac{\varepsilon^{4}}{2} \Delta_{X}+h+\varepsilon^{4} D(\varepsilon)
$$

The term $\varepsilon^{4} D(\varepsilon)$ plays the uninteresting role of a regular perturbation because it 
is relatively bounded with respect to $h$. The interesting mathematics arises from the interplay of $h$ and $-\varepsilon^{4} / 2 \Delta_{X}$, which involves a singular perturbation problem.

We will henceforth use the spherical coordinates $(R, \theta, \varphi)$ to represent the vector $X$. For each $\theta$ and $\varphi$, we let $\mathscr{R}_{1}(\theta, \varphi)$ be the rotation of $\mathbb{R}^{3}$ given by the matrix

$$
\mathscr{R}_{1}(\theta, \varphi)=\left(\begin{array}{ccc}
\cos \theta \cos \varphi & -\sin \varphi & \sin \theta \cos \varphi \\
\cos \theta \sin \varphi & \cos \varphi & \sin \theta \sin \varphi \\
-\sin \theta & 0 & \cos \theta
\end{array}\right)
$$

$\mathscr{R}_{1}(\theta, \varphi)$ maps the vector $\left(\begin{array}{l}0 \\ 0 \\ 1\end{array}\right)$ into the unit vector in the $(R, \theta, \varphi)$ direction. We define a new coordinate system $\left(\xi_{1}, \xi_{2}, \ldots, \xi_{N-2}\right)$ for the electrons that "rotates with the nuclei" by setting

$$
\xi_{j}=\left[\mathscr{R}_{1}(\theta, \varphi)\right]^{-1} \zeta_{j}
$$

Next, we define $\mathscr{R}_{2}(\gamma)$ to be the rotation

$$
\mathscr{R}_{2}(\gamma)=\left(\begin{array}{ccc}
\cos \gamma & -\sin \gamma & 0 \\
\sin \gamma & \cos \gamma & 0 \\
0 & 0 & 1
\end{array}\right)
$$

For generic $\xi_{1}$, we choose the angle $\gamma(\bmod 2 \pi)$ so that the second component of $\eta_{1}=\left[\mathscr{R}_{2}(\gamma)\right]^{-1} \xi_{1}$ is zero and the first component of $\eta_{1}$ is positive. We set $\eta_{j}=\left[\mathscr{R}_{2}(\gamma)\right]^{-1} \xi_{j}$. We let $\rho$ and $z^{\prime}$ denote the first and third components of $\eta_{1}$, respectively. At this point we can use the $3 N-3$ coordinates $\left(R, \theta, \varphi, \gamma, \rho, z^{\prime}\right.$, $\left.\eta_{2}, \eta_{3}, \ldots, \eta_{N-2}\right)$. The values of $R, \theta$, and $\varphi$ specify the spherical coordinates of the internuclear vector $X$; the (rotated) cylindrical coordinates $\gamma, \rho$, and $z^{\prime}$ specify the position of the center of mass of the electrons with reference to the internuclear axis.

In addition to rotating the electron coordinates, we will also have to dilate them. This dilation is motivated by $[3,8]$, where analyticity of the electron energy in $R$ was first proved. Given any positive value of the internuclear distance $R$, we define $\alpha=\rho / R, \beta=z^{\prime} / R$, and $\tau_{j}=\eta_{j} / R$. Our final coordinate system is given by $\left(R, \theta, \varphi, \gamma, \alpha, \beta, \tau_{2}, \tau_{3}, \ldots, \tau_{N-2}\right)$.

In this coordinate system, the electron hamiltonian $h(R, \theta, \varphi)$ has a particularly nice representation:

$$
\begin{aligned}
h(R, \theta, \varphi)= & R^{-2}\left[-\frac{1}{2 \mu_{1}}\left(\frac{\partial^{2}}{\partial \alpha^{2}}+\frac{1}{\alpha} \frac{\partial}{\partial \alpha}+\frac{1}{\alpha^{2}} \frac{\partial^{2}}{\partial \gamma^{2}}+\frac{\partial^{2}}{\partial \beta^{2}}\right)\right. \\
& \left.-\sum_{j=2}^{N-2} \frac{1}{2 \mu_{j}} \Delta_{\tau_{j}}\right]+R^{-1} W\left(\alpha, \beta, \tau_{2}, \tau_{3}, \ldots, \tau_{N-2}\right),
\end{aligned}
$$

where the potential function $W\left(\alpha, \beta, \tau_{2}, \tau_{3}, \ldots, \tau_{N-2}\right)$ is relatively bounded with respect to the kinetic energy term, with relative bound zero, and has no dependence on $R, \theta, \varphi$, or $\gamma$. In these coordinates, $h(R, \theta, \varphi)$ is independent of $\theta$ and $\varphi$, and depends on $R$ via a multiplication and a coupling constant. It is thus analytic in 
$R$ for $R>0$. In addition, $h(R, \theta, \varphi)$ commutes with $L_{z^{\prime}}=-i \partial / \partial \gamma$, the component of the total electron angular momentum about the internuclear axis.

Obtaining a representation in which $h(R, \theta, \varphi)$ depends smoothly on $R, \theta$, and $\varphi$ is the crucial step in extending the results of [15]. However, this causes a significant uglification because the nuclear kinetic energy term in $H$ is very complicated.

The eigenvalues $\Lambda$ of $L_{z^{\prime}}$ are clearly $0, \pm 1, \pm 2, \ldots$ The eigenvalues of $h(R, \theta, \varphi)$ that correspond to $\Lambda \neq 0$ must be doubly degenerate because $L_{z^{\prime}}=\Lambda$ and $L_{z^{\prime}}=-\Lambda$ give rise to the same eigenvalues for $h(R, \theta, \varphi)$. Because $L_{z^{\prime}}$ does not commute with the full Hamiltonian $H$, we will have to deal directly with this degeneracy. Of course, the eigenvalues of $h(R, \theta, \varphi)$ that correspond to $\Lambda=0$ are generically nondegenerate.

With a slight abuse of notation, we define the operator

$$
\begin{aligned}
h(R, \pm \Lambda)= & R^{-2}\left[-\frac{1}{2 \mu_{1}}\left(\frac{\partial^{2}}{\partial \alpha^{2}}+\frac{1}{\alpha} \frac{\partial}{\partial \alpha}+\frac{\Lambda^{2}}{\alpha^{2}}+\frac{\partial^{2}}{\partial \beta^{2}}\right)-\sum_{j=2}^{N-2} \frac{1}{2 \mu_{j}} \Delta_{\tau_{j}}\right] \\
& +R^{-1} W\left(\alpha, \beta, \tau_{2}, \tau_{3}, \ldots, \tau_{N-2}\right)
\end{aligned}
$$

on $L^{2}\left(\alpha / R^{3 N-7} d \alpha d \beta d^{3 N-9} \tau\right)$. We assume that $h(R, \pm \Lambda)$ has an isolated nondegenerate eigenvalue $E(R)$ for $R$ in some open neighborhood $U$ of some value $R_{0}>0$. In addition, we assume that $0 \notin U$, and that $E(R)$ has a local minimum at $R_{0}$ with $E^{\prime \prime}\left(R_{0}\right)$ strictly positive. We can then choose a normalized eigenfunction $\Phi\left(R, \alpha, \beta, \tau_{2}, \ldots, \tau_{N-2}\right)$ that is real valued for $R \in U$ and analytic in $R$ for $R$ in a complex neighborhood of $U$, so that

$$
h(R, \pm \Lambda) \Phi(R)=E(R) \Phi(R) .
$$

With this notation, we can now state our main result for the case $\Lambda=0$ :

Theorem 2.1. Assume the situation described above with $\Lambda=0$. Choose a non-negative integer $j$, and an integer $m$, with $-j \leqq m \leqq j$ to specify the total molecular angular momentum and its z component, respectively. Choose a non-negative integer $n$ to specify the vibrational state for the nuclei. Then given an arbitrary $K$, there exist quasimode energies

$$
\mathscr{E}_{n, j, m, \Lambda}(\varepsilon)=\sum_{k=0}^{K} \varepsilon^{k} E_{n, j, m, \Lambda, k}
$$

and quasimodes

$$
\Psi_{\varepsilon, n, j, m, \Lambda}\left(R, \theta, \varphi, \gamma, \alpha, \beta, \tau_{2}, \ldots, \tau_{N-2}\right)=\sum_{k=0}^{K} \varepsilon^{k} \Psi_{\varepsilon, n, j, m, \Lambda, k}\left(R, \theta, \varphi, \alpha, \beta, \gamma, \tau_{2}, \ldots, \tau_{N-2}\right),
$$

so that

$$
\begin{aligned}
& \| H(\varepsilon) \Psi_{\varepsilon, n, j, m, \Lambda}\left(R, \theta, \varphi, \gamma, \alpha, \beta, \tau_{2}, \ldots, \tau_{N-2}\right) \\
& \quad-\mathscr{E}_{n, j . m . \Lambda}(\varepsilon) \Psi_{\varepsilon, n, j, m, \Lambda}\left(R, \theta, \varphi, \gamma, \alpha, \beta, \tau_{2}, \ldots, \tau_{N-2}\right) \| \leqq C_{n, j, m, K} \varepsilon^{K+1} .
\end{aligned}
$$

The numbers $E_{n, 1, m, \Lambda ; 1}, E_{n, J, m, \Lambda, 3}$, and $E_{n, l, m, \Lambda ; 5}$ are always zero. $E_{n, j, m, \Lambda ; 0}=E\left(R_{0}\right)$ is the electronic contribution to the energy, and $E_{n, 1, m, \Lambda, 2}=(n+1 / 2)\left[E^{\prime \prime}\left(R_{0}\right)\right]^{1 / 2}$ is the harmonic approximation to the nuclear vibrational energy. The formula for $E_{n,, m, \Lambda ; 4}$ 
is given in the remarks below. The zero ${ }^{\text {th }}$ order term in the quasimode is

$$
\begin{aligned}
& \Psi_{\varepsilon, n, j, m, \Lambda ; 0}\left(R, \theta, \varphi, \gamma, \alpha, \beta, \tau_{2}, \ldots, \tau_{N-2}\right) \\
& =N_{n} R^{-1} H_{n}\left(\frac{\left[E^{\prime \prime}\left(R_{0}\right)\right]^{1 / 2}\left(R-R_{0}\right)}{\varepsilon}\right) e^{-E^{\prime \prime}\left(R_{0}\right)\left(R-R_{0}\right)^{2} / 2 \varepsilon^{2}} \\
& \quad \times Y_{j, m}(\theta, \varphi) \Phi\left(R, \alpha, \beta, \tau_{2}, \ldots, \tau_{N-2}\right),
\end{aligned}
$$

where $N_{n}$ is a normalization constant and $H_{n}$ is the $n^{\text {th }}$ degree Hermite polynomial.

Remarks. 1. There may be several different choices of $E(R)$ and $R_{0}$, depending on the details of the electronic hamiltonian $h(R, 0)$.

2. If $\mathscr{E}_{n, j, m, \Lambda}(\varepsilon)$ lies below the essential spectrum of $H(\varepsilon)$ for all small $\varepsilon$, then it asymptotically corresponds to an eigenvalue of $H(\varepsilon)$.

3. It is reasonable to conjecture that the quasimodes of the theorem whose energies lie in the essential spectrum of $H(\varepsilon)$ correspond to resonances of the molecule.

4. If $E(R)$ is chosen to be the ground state energy of $h(R, 0)$, and if $E(R)$ has a global minimum at $R_{0}$ with $E^{\prime \prime}\left(R_{0}\right)>0$, then the asymptotics of the lowest lying eigenvalues of $H(\varepsilon)$ are completely described by the corresponding $\mathscr{E}_{n, j, m .0}(\varepsilon)$, i.e., there are no other low lying eigenvalue asymptotics. This is a result of [5-7].

5. The formula for $E_{n, j, \Lambda ; 4}$ depends on the electronic cigenfunction $\Phi$, but we can still give a fairly explicit formula for it. Explicitly,

$$
E_{n, j, m, \Lambda, 4}=a_{j}+b+c_{n}, \quad \text { where } a_{j}=j(j+1) / 2\left(R_{0}\right)^{2}
$$

is the dominant term in the angular momentum dependence of the molecule's energy;

$$
b=\left\langle\Phi(R, \theta, \varphi, \cdot),\left[-\frac{1}{2} \Delta_{X}+D_{0}\right] \Phi(R, \theta, \varphi, \cdot)\right\rangle_{L^{2}(d r)}
$$

is the lowest order nontrivial coupling of the electronic and nuclear motions $\left(D_{0}\right.$ is the constant term in the power series in $\varepsilon^{4}$ for $D(\varepsilon)$ ); and

$$
c_{n}=-\left[\frac{E^{\prime \prime \prime}\left(R_{0}\right)}{E^{\prime \prime}\left(R_{0}\right)}\right]^{2}\left(\frac{11}{288}+\frac{5 n(n+1)}{48}\right)+\frac{E^{\prime \prime \prime \prime}\left(R_{0}\right)}{E^{\prime \prime}\left(R_{0}\right)}\left(\frac{1}{32}+\frac{n(n+1)}{16}\right)
$$

is the lowest anharmonic correction to the nuclear vibrational energy.

6. Through fourth order, $\mathscr{E}_{n, j, m, \Lambda}(\varepsilon)$ has no $m$-dependence, so to that order, each quasimode energy corresponds to $2 j+1$ orthogonal quasimodes if $\Lambda=0$. Except for degeneracies due to symmetries (e.g., identical nuclei), we expect this degeneracy to be broken at sixth order. Note that one can use non-degenerate perturbation theory because $J_{z}$ commutes with the Hamiltonian, and the states of different $m$ are not coupled.

The case of $\Lambda \neq 0$ is not substantially different, except that one must use degenerate perturbation theory and replace the familiar spherical harmonics $Y_{j . m}(\theta, \varphi)$ with the less familiar eigenfunctions $\mathscr{D}_{m . \Lambda}^{(j)}(\theta, \varphi, \gamma)$ of the total angular momentum operator for the molecule. These eigenfunctions are described in [11], and have the form $\mathscr{Z}_{m, \Lambda}^{(j)}(\theta, \varphi, \gamma)=e^{\operatorname{im} \varphi} d_{m . \Lambda}^{(j)}(\theta) e^{i \Lambda \gamma}$, where $d_{m, \Lambda}^{(j)}(\theta)$ can be explicitly expressed in terms of trigonometric functions. The following theorem summarizes our results in the case $\Lambda \neq 0$ : 
Theorem 2.2. Assume the situation described above with $\Lambda \neq 0$. Choose an integer $j \geqq|\Lambda|$, and an integer $m$, with $-j \leqq m \leqq j$. Choose a non-negative integer $n$. Then given an arbitrary $K$, there exist two quasimode energies

$$
\mathscr{E}_{n, l, m,|\lambda|, \pm}(\varepsilon)=\sum_{k=0}^{K} \varepsilon^{k} E_{n, J, m\}, \Lambda \mid \pm h}
$$

and two orthogonal quasimodes

$$
\begin{aligned}
& \Psi_{\varepsilon, n, \gamma, m, \mid \Lambda, \pm}\left(R, \theta, \varphi, \gamma, \alpha, \beta, \tau_{2}, \ldots, \tau_{N-2}\right) \\
& \quad=\sum_{k=0}^{K} \varepsilon^{k} \Psi_{t, n, j, m,|,| A \mid, \pm k}\left(R, \theta, \varphi, \gamma, \alpha, \beta, \gamma, \tau_{2}, \ldots, \tau_{N-2}\right),
\end{aligned}
$$

so that

$$
\begin{aligned}
& \| H(\varepsilon) \Psi_{\tau, n, j, m, A\}, \pm}\left(R, \theta, \varphi, \gamma, \alpha, \beta, \tau_{2}, \ldots, \tau_{N-2}\right) \\
& -\mathscr{E}_{n, j, m,|\Lambda| \pm}(\varepsilon) \Psi_{\varepsilon, n, j, m,|\Lambda|, \pm}\left(R, \theta, \varphi, \gamma, \alpha, \beta, \tau_{2}, \ldots, \tau_{N-2}\right) \| \leqq C_{n, l, m, K} \varepsilon^{K+1} .
\end{aligned}
$$

The numbers $E_{n,|m,| \Lambda \mid \pm, 1}, E_{n,, m,|\Lambda| \pm 3}$, and $E_{n, 1, m,|\Lambda|, \pm, 5}$ are always zero. $E_{n, 1, m,|\Lambda|, \pm ; 0}=E\left(R_{0}\right)$ is the electronic contribution to the energy, and $E_{n,, m,|A| \pm, 2}=(n+1 / 2)\left[E^{\prime \prime}\left(R_{0}\right)\right]^{1 / 2}$ is the harmonic approximation to the nuclear vibrational energy. The formula for $E_{n, j, m,|1|, \pm ; 4}$ is given in Remark 5 above. The zeroth order term in the quasimode is

$$
\begin{aligned}
& \Psi_{\varepsilon, n, j, m, \mid 1 ; \pm ; 0}\left(R, \theta, \varphi, \gamma, \alpha, \beta, \tau_{2}, \ldots, \tau_{N-2}\right) \\
& =R^{-1} H_{n}\left(\frac{\left[E^{\prime \prime}\left(R_{0}\right)\right]^{1 / 2}\left(R-R_{0}\right)}{\varepsilon}\right) e^{-E^{\prime \prime}\left(R_{0}\right)\left(R-R_{0}\right)^{2} / 2 t^{2}} \Phi\left(R, \alpha, \beta, \tau_{2}, \ldots, \tau_{N-2}\right) \\
& \times\left[N_{n,+}^{ \pm}+\mathscr{D}_{m,|\Lambda|}^{(j)}(\theta, \varphi, \gamma)+N_{n, D_{m,-\mid \Lambda i}^{ \pm}}^{(j)}(\theta, \varphi, \gamma)\right],
\end{aligned}
$$

where $N_{n, \pm}^{ \pm}$are constants and $H_{n}$ is the $n^{\text {th }}$ degree Hermite polynomial.

Remark. Remarks 1,2,3, and 5 following Theorem 2.1 also apply to Theorem 2.2. Remark 4 does not apply to Theorem 2.2 because the ground state always has $j=0$ (we are ignoring spin and statistics). Remark 6 applies to Theorem 2.2, except for the following modifications: There are $4 j+2$ degenerate states at fourth order instead of $2 j+1$. For each $m$ one must use degenerate perturbation theory on the pairs of states with $L_{z^{\prime}}= \pm \Lambda$. These states are necessarily doubly degenerate through at least order $\varepsilon^{8|A|}$ because of selection rules that are obeyed by the $J \cdot L$ term that is responsible for breaking the degeneracy ( $L$ is the total electronic angular momentum). See Sect. 3 [17, pp. 121-122], or [18, p. 337]. This high order ( $\Lambda$-doubling) splitting is very small unless $|\Lambda|=1$. We expect all degeneracies (except for the $A$-doubling and any degeneracy due to symmetries) to be broken at sixth order.

\section{Formal Derivation of the Expansion}

In this section we will formally derive the results of Sect. 2. The basic ideas are the use of the particular coordinate systems defined in Sect. 2 and the "method of multiple scales" in the variable $R$. 
The method of multiple scales was already used in $[14,15]$ to separate the adiabatic and semiclassical effects, so we will not discuss it at length here. Physically, the idea is that the (semiclassical) nuclear vibrations occur on a length scale of order $\varepsilon$ in $R$, and the (adiabatic) electronic states are sensitive to nuclear motions on the order of 1 in $R$. So it is advantageous to introduce two variables $x=R$ and $y=\left(R-R_{0}\right) / \varepsilon$, and treat them as though they were independent. I.e., we will search for functions $\Psi_{\varepsilon, n, j, m, \Lambda}\left(x, y, \theta, \varphi, \gamma, \alpha, \beta, \tau_{2}, \ldots, \tau_{N-2}\right)$, so that $\Psi_{\varepsilon, n, j, m, \Lambda}\left(R,\left(R-R_{0}\right) / \varepsilon, \theta, \varphi, \gamma, \mu, \beta, \tau_{2}, \ldots, \tau_{N-2}\right)$ will satisfy the Schrödinger equation. This use of the two variables $x$ and $y$ allows one to separate variables in the low orders of the approximation, and provides a clean formalism for separating the high order semiclassical and adiabatic effects. Without this separation, the analysis is prohibitively complicated.

We have split this section into two parts. The first part deals with the simpler case of one electron, and is very explicit. The second part treats the many electron case, but does not contain explicit formulas. We have done this to make the details clear in a simpler situation, and to describe the general case without explicitly writing out all the messy formulas.

3A. The One Electron Case. We first consider the case $\Lambda=0$. To simplify the radial Laplacian, we make the standard change of dependent variable and concentrate on $\psi_{\varepsilon}(R, \theta, \varphi, \gamma, \alpha, \beta)=R \Psi_{\varepsilon}(R, \theta, \varphi, \gamma, \alpha, \beta)$. where $\Psi_{\varepsilon}$ is the eigenfunction of $H(\varepsilon)$. In the coordinate system of Sect. 2 , the Hamiltonian has the form

$$
\begin{aligned}
H(\varepsilon)= & \frac{\varepsilon^{4}}{2}\left[-\frac{\partial^{2}}{\partial R^{2}}-\frac{\alpha^{2}}{R^{2}} \frac{\partial^{2}}{\partial \alpha^{2}}-\frac{\beta^{2}}{R^{2}} \frac{\partial^{2}}{\partial \beta^{2}}+2 \frac{\alpha}{R} \frac{\partial^{2}}{\partial \alpha \partial R}+2 \frac{\beta}{R} \frac{\partial^{2}}{\partial \beta \partial R}\right. \\
& \left.-2 \frac{\alpha \beta}{R^{2}} \frac{\partial^{2}}{\partial \alpha \partial \beta}-2 \frac{\alpha}{R^{2}} \frac{\partial}{\partial \alpha}-2 \frac{\beta}{R^{2}} \frac{\partial}{\partial \beta}+\frac{J^{2}-2 L \cdot J+L^{2}}{R^{2}}\right]+h(R)+\varepsilon^{4} D(\varepsilon),
\end{aligned}
$$

where

$$
\begin{aligned}
J^{2}= & -\frac{\partial^{2}}{\partial \theta^{2}}-\cot \theta \frac{\partial}{\partial \theta}-\frac{1}{\sin ^{2} \theta}\left(\frac{\partial^{2}}{\partial \varphi^{2}}+\frac{\partial^{2}}{\partial \gamma^{2}}\right)+\frac{2 \cos \theta}{\sin ^{2} \theta} \frac{\partial^{2}}{\partial \varphi \partial \gamma^{\prime}} \\
L \cdot J= & \left(\alpha \sin \gamma \frac{\partial}{\partial \beta}-\beta \sin \gamma \frac{\partial}{\partial \alpha}-\frac{\beta}{\alpha} \cos \gamma \frac{\partial}{\partial \gamma}\right)\left(\frac{1}{\sin \theta} \frac{\partial}{\partial \varphi}-\cot \theta \frac{\partial}{\partial \gamma}\right) \\
& -\left(\alpha \cos \gamma \frac{\partial}{\partial \beta}-\beta \cos \gamma \frac{\partial}{\partial \alpha}+\frac{\beta}{\alpha} \sin \gamma \frac{\partial}{\partial \gamma}\right) \frac{\partial}{\partial \theta}-\frac{\partial^{2}}{\partial \gamma^{2}}, \\
L^{2}= & -\alpha^{2} \frac{\partial^{2}}{\partial \beta^{2}}-\beta^{2} \frac{\partial^{2}}{\partial \alpha^{2}}-\frac{\beta^{2}}{\alpha^{2}} \frac{\partial^{2}}{\partial \gamma^{2}}+2 \alpha \beta \frac{\partial^{2}}{\partial \alpha \partial \beta}+\left(\alpha-\frac{\beta^{2}}{\alpha}\right) \frac{\partial}{\partial \alpha}+2 \beta \frac{\partial}{\partial \beta}-\frac{\partial^{2}}{\partial \gamma^{2}}, \\
h(R)= & -\frac{R^{-2}}{2 m}\left[\frac{\partial^{2}}{\partial \alpha^{2}}+\frac{1}{\alpha} \frac{\partial}{\partial \alpha}+\frac{1}{\alpha^{2}} \frac{\partial^{2}}{\partial \gamma^{2}}+\frac{\partial^{2}}{\partial \beta^{2}}\right] \\
& +\frac{Z_{1} Z_{2}}{R}-\frac{Z_{2}}{R\left[\alpha^{2}+(\beta+\delta)^{2}\right]^{1 / 2}}-\frac{Z_{2}}{R\left[\alpha^{2}+(\beta-(1-\delta))^{2}\right]^{1 / 2}},
\end{aligned}
$$

(here, $\delta$ is the nuclear mass ratio $\delta=M_{2} /\left(M_{1}+M_{2}\right)$ ), and 


$$
\varepsilon^{4} D(\varepsilon)=\frac{1}{2 R^{2}}\left(\frac{1}{v(\varepsilon)}-\frac{1}{\mu(\varepsilon)}\right)\left[\frac{\partial^{2}}{\partial \alpha^{2}}+\frac{1}{\alpha} \frac{\partial}{\partial \alpha}+\frac{1}{\alpha^{2}} \frac{\partial^{2}}{\partial \gamma^{2}}+\frac{\partial^{2}}{\partial \beta^{2}}\right]
$$

We choose a non-degenerate electron energy $E(R)$ and choose angular momentum quantum numbers $j$ and $m$, corresponding to $J^{2}$ and $J_{z}=-i(\partial / \partial \varphi)$. We next formally change variables to do the multiple scales to obtain the equation satisfied by the function $\psi_{\varepsilon}(x, y, \theta, \varphi, \gamma, \alpha, \beta)$. The variable $R$ is replaced by $x$ in some situations and by $\left[R_{0}+\varepsilon y\right]$ in others; The operator $\partial / \partial R$ is replaced by $((\partial / \partial x)+$ $\left.\varepsilon^{-1}(\partial / \partial y)\right)$. As in [15], we also have to introduce some operators $T_{k}$ whose purpose is to change $x$ dependence into $y$ dependence. These operators do the bookkeeping associated with the fact that $x$ and $y$ are not actually independent. Without these operators one could not treat $x$ and $y$ as though they were independent. The choice of the $T_{k}$ 's also provides a uniqueness condition in the expansion.

The equation satisfied by $\psi_{\varepsilon}(x, y, \theta, \varphi, \gamma, \alpha, \beta)$ is the following:

$$
\begin{aligned}
\left(\frac{\varepsilon^{4}}{2}[\right. & -\frac{\partial^{2}}{\partial x^{2}}-\frac{\alpha^{2}}{x^{2}} \frac{\partial^{2}}{\partial \alpha^{2}}-\frac{\beta^{2}}{x^{2}} \frac{\partial^{2}}{\partial \beta^{2}}+2 \frac{\alpha}{x} \frac{\partial^{2}}{\partial \alpha \partial x}+2 \frac{\beta}{x} \frac{\partial^{2}}{\partial \beta \partial x}-2 \frac{\alpha \beta}{x^{2}} \frac{\partial^{2}}{\partial \alpha \partial \beta} \\
& \left.-2 \frac{\alpha}{x^{2}} \frac{\partial}{\partial \alpha}-2 \frac{\beta}{x^{2}} \frac{\partial}{\partial \beta}+\frac{J^{2}-2 L \cdot J+L^{2}}{\left[R_{0}+\varepsilon y\right]^{2}}\right]+\varepsilon^{3}\left[-\frac{\partial^{2}}{\partial x \partial y}+\frac{\alpha}{x} \frac{\partial^{2}}{\partial \alpha \partial y}+\frac{\beta}{x} \frac{\partial^{2}}{\partial \beta \partial y}\right] \\
& -\frac{\varepsilon^{2}}{2} \frac{\partial^{2}}{\partial y^{2}}+E\left(R_{0}+\varepsilon y\right)-E(x)+h(x)+\varepsilon^{4} D(\varepsilon) \\
& \left.+\sum_{k=4}^{\infty} \varepsilon^{k}\left[T_{k}\left(R_{0}+\varepsilon y\right)-T_{k}(x)\right]\right) \psi_{\varepsilon}(x, y, \theta, \varphi, \gamma, \alpha, \beta) \\
= & \mathscr{E}(\varepsilon) \psi_{\varepsilon}(x, y, \theta, \varphi, \gamma, \alpha, \beta),
\end{aligned}
$$

where $J^{2}, L \cdot J, L^{2}$, and $h(\cdot)$ are as above. It is trivial to check that a solution to (3.1) gives rise to a solution to the original Schrödinger equation, independent of the choice of the $T_{k}$ 's. We will choose $T_{k}(\cdot)$ to be certain multiplication operators. These choices will be made so that certain functions in the expansion will be independent of $x$. This, in turn, will impose a uniqueness condition on the expansion. We note that changing $T_{k}(\cdot)$ by an additive constant does not alter Eq. (3.1).

We now make an ansatz:

$$
\psi_{\varepsilon}=\left(\psi_{0}+\varepsilon \psi_{1}+\varepsilon^{2} \psi_{2}+\cdots\right) F(x)
$$

and

$$
\mathscr{E}(\varepsilon)=\mathscr{E}_{0}+\varepsilon \mathscr{E}_{1}+\varepsilon^{2} \mathscr{E}_{2}+\cdots .
$$

Here $F(\mathrm{x})$ is a $C^{\infty}$ function of compact support that is identically 1 on an open neighborhood of $x=R_{0}$, and has support inside the set $U$, where $E(x)$ is non-degenerate.

Remark. The reader who is not interested in the formal computations but not a detailed proof is encouraged to ignore the factor $F(x)$ in this ansatz, and think of the open set $U$ as all of $(0, \infty)$. The factor provides some uniformity that is required for the rigorous proof of Sect. 4. As in [15], we will ignore terms containing 
derivatives of $F$ that occur below, because they do not contribute at any finite order of the expansion. See Sect. 4.

We now determine the $\psi_{n}$. Since $F(x)=0$ for $x \notin U$, we arbitrarily set $\psi_{n}(x, y, \theta, \varphi, \gamma, \alpha, \beta)=0$ for all $x \notin U$. For $x \in U$ we substitute the expressions (3.2) into Eq. (3.1) and expand all $\varepsilon$ dependence in its Taylor series in powers of $\varepsilon$. Then we multiply everything out and equate coefficients of like powers of $\varepsilon$ on the two sides of the equation.

The zeroth order terms force us to take

$$
\left[h(x)-E(x)+E\left(R_{0}\right)\right] \psi_{0}=\mathscr{E}_{0} \psi_{0} .
$$

Since this is to be true for all $x \in U$, we are forced to take

$$
\mathscr{E}_{0}=E\left(R_{0}\right)
$$

and

$$
\psi_{0}(x, y, \theta, \varphi, \gamma, \alpha, \beta)=h_{0}(x, y, \theta, \varphi) \Phi(x, \gamma, \alpha, \beta) .
$$

Because we have chosen $\Lambda=0, \Phi$ has no $\gamma$ dependence. In addition, we are simultaneously diagonalizing $J^{2}$ and $J_{z}$, so we have (with a slight abuse of notation)

$$
\psi_{0}(x, y, \theta, \varphi, \gamma, \alpha, \beta)=g_{0}(x, y) Y_{j, m}(\theta, \varphi) \Phi(x, \alpha, \beta) .
$$

At this point, we will insist that $g_{0}$ have no $x$ dependence. To see that we can impose this condition, suppose that $g_{0}$ had some $x$ dependence. Then, in our final answer, $g_{0}(x, y)$ would be equivalent to $g_{0}\left(R_{0}+\varepsilon y, y\right)$. We could then expand this final expression in Taylor series in $\varepsilon$ and redefine $g_{0}$ to be the constant term of the expansion. The higher terms of the Taylor expansion could be incorporated in the higher $\psi_{n}$ 's. Thus, we can assume

$$
\psi_{0}(x, y, \theta, \varphi, \gamma, \alpha, \beta)=f_{0}(y) Y_{j, m}(\theta, \varphi) \Phi(x, \alpha, \beta),
$$

where $f_{0}$ is so far arbitrary.

At every order of the expansion, we will impose a similar condition, namely that a function $g_{n}(x, y)=f_{n}(y)$. By forcing these conditions, we essentially determine the operators $T_{k}(x)$. If the terms $T_{k}(x)-T_{k}\left(R_{0}+\varepsilon y\right)$ were not present in Eq. (3.1), we would not be able to consistently impose this condition.

The first order terms force us to take

$$
\left[h(x)-E(x)+E\left(R_{0}\right)\right] \psi_{1}+E^{\prime}\left(R_{0}\right) y \psi_{0}=\mathscr{E}_{0} \psi_{1}+\mathscr{E}_{1} \psi_{0} .
$$

Since this is to hold for all $y$ and $x \in U$, we must have

$$
\mathscr{E}_{1}=E^{\prime}\left(R_{0}\right)=0
$$

and

$$
\psi_{1}(x, y, \theta, \varphi, \gamma, \alpha, \beta)=h_{1}(x, y, \theta, \varphi) \Phi(x, \alpha, \beta) .
$$

Once again, the $\theta, \varphi$, and $\gamma$ dependence is determined, so

$$
\psi_{1}(x, y, \theta, \varphi, \gamma, \alpha, \beta)=g_{1}(x, y) Y_{j, m}(\theta, \varphi) \Phi(x, \alpha, \beta) .
$$

We arbitrarily choose $g_{1}(x, y)=f_{1}(y)$, which we may do for reasons that were 
explained above. So, we have

$$
\psi_{1}(x, y, \theta, \varphi, \gamma, \alpha, \beta)=f_{1}(y) Y_{j, m}(\theta, \varphi) \Phi(x, \alpha, \beta),
$$

where $f_{1}$ is so far arbitrary.

The second order terms require

$$
[h(x)-E(x)] \psi_{2}+\left[-\frac{1}{2} \frac{\partial^{2}}{\partial y^{2}}+\frac{1}{2} E^{\prime \prime}\left(R_{0}\right) y^{2}\right] \psi_{0}=\mathscr{E}_{2} \psi_{0} .
$$

To satisfy this equation, we separately consider those terms that are multiples of $\Phi$ and those that are orthogonal to $\Phi$, as in [15]. We recall that we have assumed $E^{\prime \prime}\left(R_{0}\right)>0$. If we let $w=y\left(E^{\prime \prime}\left(R_{0}\right)\right)^{1 / 2}$, then the second order terms require

$$
\begin{aligned}
\mathscr{E}_{2} & =\left(n+\frac{1}{2}\right)\left(E^{\prime \prime}\left(R_{0}\right)\right)^{1 / 2}, \\
\psi_{0}(x, y, \theta, \varphi, \gamma, \alpha, \beta) & =H_{n}(w) e^{-w^{2} / 2} Y_{j, m}(\theta, \varphi) \Phi(x, \alpha, \beta),
\end{aligned}
$$

and

$$
\psi_{2}(x, y, \theta, \varphi, \gamma, \alpha, \beta)=h_{2}(x, y, \theta, \varphi) \Phi(x, \alpha, \beta),
$$

where $H_{n}$ denotes the $n^{\text {th }}$ degree Hermite polynomial. By the angular momentum considerations and the $x$-independence condition,

$$
\psi_{2}(x, y, \theta, \varphi, \gamma, \alpha, \beta)=f_{2}(y) Y_{j, m}(\theta, \varphi) \Phi(x, \alpha, \beta),
$$

Remark. We will not worry about normalizing our quasimodes. If we were to normalize them, the expression for $\psi_{0}$, above, would be multiplied by a constant.

We now consider the third order terms. There is one third order term that contains a derivative of $F$. It makes no contribution to the expansion at any finite order (see Sect. 4), so we will ignore it here. The remaining terms require

$$
\begin{aligned}
& {[h(x)-E(x)] \psi_{3}+\left[-\frac{1}{2} \frac{\partial^{2}}{\partial y^{2}}+\frac{1}{2} E^{\prime \prime}\left(R_{0}\right) y^{2}-\mathscr{E}_{2}\right] \psi_{1}+\frac{1}{6} E^{\prime \prime \prime}\left(R_{0}\right) y^{3} \psi_{0}} \\
& -\left[\frac{\partial^{2}}{\partial x \partial y}-\frac{\alpha}{x} \frac{\partial^{2}}{\partial \alpha \partial y}-\frac{\beta}{x} \frac{\partial^{2}}{\partial \beta \partial y}\right] \psi_{0}=\mathscr{E}_{3} \psi_{0} .
\end{aligned}
$$

To satisfy this equation we introduce some new notation. We break up $\psi_{n}$ as

where

$$
\psi_{n}=\psi_{n}^{\perp}+\psi_{n}^{\perp}+\psi_{n}^{\prime}
$$

$$
\begin{aligned}
& \psi_{n}^{\perp} \text { is orthogonal to } \Phi(x, \gamma, \alpha, \beta) \text { in } L^{2}\left(\frac{\alpha}{x^{2}} d \gamma d \alpha d \beta\right) \\
& \psi_{n}^{\perp} \text { is a multiple of } \Phi(x, \gamma, \alpha, \beta) \text {, but orthogonal to } \\
& \qquad H_{n}(w) e^{-w^{2} / 2} \text { in } L^{2}(d y) \text {; and } \\
& \psi_{n} \text { is a multiple of } H_{n}(w) e^{-w^{2} / 2} \Phi(x, \gamma, \alpha, \beta) .
\end{aligned}
$$

With this notation, we can now satisfy Eq. (3.3) by looking at the components in the various directions in the Hilbert space:

The components on the two sides of Eq. (3.3) that are $x, \theta$, and $\varphi$ dependent 
multiples of $H_{n}(w) e^{-w^{2} / 2} \Phi(x, \alpha, \beta)$ must be equal. From this, total angular momentum diagonalization, and our $x$-independence condition, we obtain

and

$$
\mathscr{E}_{3}=0 \text {, }
$$

$$
\psi_{1}=c_{1} H_{n}(z) e^{-z^{2} / 2} Y_{j, m}(\theta, \varphi) \Phi(x, \alpha, \beta),
$$

where $c_{1}$ is an arbitrary constant. Nothing is gained by choosing $c_{1}$ to be non-zero, except for keeping the normalization of the wave function (about which we do not care), so we arbitrarily choose $c_{1}=0$. Thus,

$$
\psi_{1}^{\prime \prime}=0 \text {. }
$$

In fact, to impose uniqueness for the higher order terms, we impose the condition that all such secular terms, $\psi_{n}(n \geqq 1)$, be set equal to zero.

The components of Eq. (3.3) that are multiples of $\Phi(x, \alpha, \beta)$, but orthogonal to $H_{n}(w) e^{-w^{2} / 2}$ must be equal on the two sides of the equation. We note that the term

$$
-\left[\frac{\partial^{2}}{\partial x \partial y}-\frac{\alpha}{x} \frac{\partial^{2}}{\partial \alpha \partial y}-\frac{\beta}{x} \frac{\partial^{2}}{\partial \beta \partial y}\right] \psi_{0}
$$

in Eq. (3.3) is orthogonal to $\Phi(x, \alpha, \beta)$ in $L^{2}\left(\left(\alpha / x^{2}\right) d \gamma d \alpha d \beta\right)$ because by changing back to the original coordinate system,

$$
\begin{aligned}
& \left\langle\Phi(x, \alpha, \beta),\left[\frac{\partial}{\partial x}-\frac{\alpha}{x} \frac{\partial}{\partial \alpha}-\frac{\beta}{x} \frac{\partial}{\partial \beta}\right] \Phi(x, \alpha, \beta)\right\rangle_{L^{2}\left(\left(\alpha / x^{2}\right) d \gamma d x d \beta\right)} \\
& =\left\langle\Phi\left(R, \zeta_{1}\right), \frac{\partial}{\partial R} \Phi\left(R, \zeta_{1}\right)\right\rangle_{L^{2}\left(d \zeta_{1}\right)} \\
& =\frac{1}{2} \frac{\partial}{\partial R}\left\langle\Phi\left(R, \zeta_{1}\right), \Phi\left(R, \zeta_{1}\right)\right\rangle_{L^{2}\left(d \zeta_{1}\right)} \\
& =0 .
\end{aligned}
$$

So,

$$
\psi_{1}{ }^{\perp}=\Phi(x, \alpha, \beta) Y_{j, m}(\theta, \varphi)\left[H_{\mathrm{osc}}-\mathscr{E}_{2}\right]_{r}^{-1}\left(-\frac{1}{6} E^{\prime \prime \prime}\left(R_{0}\right) y^{3} H_{n}(w) e^{-w^{2} / 2}\right),
$$

where $\left[H_{\mathrm{osc}}-\mathscr{E}_{2}\right]_{r}^{-1}$ denotes the inverse of the restriction of $\left[-\frac{1}{2}\left(\partial^{2} / \partial y^{2}\right)+\right.$ $\left.\frac{1}{2} E^{\prime \prime}\left(R_{0}\right) y^{2}-\mathscr{E}_{2}\right]$ to the subspace of $L^{2}(d y)$ orthogonal to $H_{n}(w) e^{-w^{2} / 2}$. We note that $\left(-\frac{1}{6} E^{\prime \prime \prime}\left(R_{0}\right) y^{3} H_{n}(w) e^{-w^{2} / 2}\right)$ belongs to this subspace because of symmetries.

The components that are orthogonal to $\Phi(x, \alpha, \beta)$ in $L^{2}\left(\left(\alpha / x^{2}\right) d \gamma d \alpha d \beta\right)$ must also be equal on the two sides of Eq. (3.3). So we have

$$
\begin{aligned}
\psi_{3}^{\perp}= & Y_{J, m}(\theta, \varphi)\left[\frac{\partial}{\partial y} H_{n}(w) e^{-w^{2} / 2}\right] \\
& \cdot\left([h(x)-E(x)]_{r}^{-1}\left[\frac{\partial \Phi}{\partial x}-\frac{\alpha}{x} \frac{\partial \Phi}{\partial \alpha}-\frac{\beta}{x} \frac{\partial \Phi}{\partial \beta}\right]\right)(x, \alpha, \beta),
\end{aligned}
$$

where $[h(x)-E(x)]_{r}^{-1}$ denotes the inverse of the restriction of $[h(x)-E(x)]$ to the subspace of $L^{2}\left(\left(\alpha / x^{2}\right) d \gamma d \alpha d \beta\right)$ orthogonal to $\Phi(x, \alpha, \beta)$. 
We now concentrate on the fourth order terms in Eq. (3.1). They require

$$
\begin{aligned}
{[h(x)} & -E(x)] \psi_{4}+\left[-\frac{1}{2} \frac{\partial^{2}}{\partial y^{2}}+\frac{1}{2} E^{\prime \prime}\left(R_{0}\right) y^{2}-\mathscr{E}_{2}\right] \psi_{2} \\
& +\frac{1}{6} E^{\prime \prime \prime}\left(R_{0}\right) y^{3} \psi_{1}+\frac{1}{24} E^{\prime \prime \prime \prime}\left(R_{0}\right) y^{4} \psi_{0} \\
& -\left[\frac{\partial^{2}}{\partial x \partial y}-\frac{\alpha}{x} \frac{\partial^{2}}{\partial \alpha \partial y}-\frac{\beta}{x} \frac{\partial^{2}}{\partial \beta \partial y}\right] \psi_{1}+A \psi_{0}+D(0) \psi_{0} \\
& +\frac{j(j+1)}{2 R_{0}^{2}} \psi_{0}-\frac{L \cdot J}{R_{0}^{2}} \psi_{0}+\frac{L^{2}}{2 R_{0}^{2}} \psi_{0}+T_{4}\left(R_{0}\right) \psi_{0}-T_{4}(x) \psi_{0} \\
= & \mathscr{E}_{4} \psi_{0},
\end{aligned}
$$

where

$$
\begin{aligned}
A= & \frac{1}{2}\left[-\frac{\partial^{2}}{\partial x^{2}}-\frac{\alpha^{2}}{x^{2}} \frac{\partial^{2}}{\partial \alpha^{2}}-\frac{\beta^{2}}{x^{2}} \frac{\partial^{2}}{\partial \beta^{2}}+2 \frac{\alpha}{x} \frac{\partial^{2}}{\partial \alpha \partial x}+2 \frac{\beta}{x} \frac{\partial^{2}}{\partial \beta \partial x}\right. \\
& \left.-2 \frac{\alpha \beta}{x^{2}} \frac{\partial^{2}}{\partial \alpha \partial \beta}-2 \frac{\alpha}{x^{2}} \frac{\partial}{\partial \alpha}-2 \frac{\beta}{x^{2}} \frac{\partial}{\partial \beta}\right] .
\end{aligned}
$$

We have again ignored terms involving derivatives of $F$ because they will be shown in Sect. 4 to not contribute at finite order. The components of Eq. (3.4) that are $x, \theta$ and $\varphi$ dependent multiples of $H_{n}(w) e^{-w^{2} / 2} \Phi(x, \gamma, \alpha, \beta)$ force us to have

$$
\begin{aligned}
& \frac{j(j+1)}{2 R_{0}^{2}} H_{n}(w) e^{-w^{2} / 2} \\
& \quad-\frac{1}{36}\left[E^{\prime \prime \prime}\left(R_{0}\right)\right]^{2} P_{y}\left(y^{3}\left[H_{\mathrm{osc}}-\mathscr{E}_{2}\right]_{r}^{-1} y^{3} H_{n}(w) e^{-w^{2} / 2}\right) \\
& \quad+\frac{1}{24} E^{\prime \prime \prime \prime}\left(R_{0}\right) P_{y}\left(y^{4} H_{n}(w) e^{-w^{2} / 2}\right) \\
& \quad+H_{n}(w) e^{-w^{2} / 2}\left\langle\Phi(x, \cdot),\left[A+\frac{L^{2}}{2 R_{0}^{2}}+D(0)\right] \Phi(x, \cdot)\right\rangle L_{L^{2}\left(\left(\alpha / x^{2}\right) d x d \beta d \gamma\right)} \\
& \quad-T_{4}(x) H_{n}(w) e^{-w^{2} / 2}+T_{4}\left(R_{0}\right) H_{n}(w) e^{-w^{2} / 2} \\
& =\mathscr{E}_{4} H_{n}(w) e^{-w^{2} / 2},
\end{aligned}
$$

where $P_{v}$ denotes the orthogonal projection in $L^{2}(d y)$ onto the subspace generated by $H_{n}(w) e^{-w^{2} / 2}$.

We note that there is no $L \cdot J$ term in Eq. (3.5). This occurs for two reasons. Let $x^{\prime}, y^{\prime}$, and $z^{\prime}$ denote the rotated coordinate axes. Then, $L_{z^{\prime}} \cdot J_{z^{\prime}} Y_{j, m} \Phi=0$, because we have chosen $\Lambda=0$. The remaining $L \cdot J$ terms do not contribute because of selection rules $([17$, pp. 121-122] or $[18$, p. 337]) that can be derived from the angular momentum commutation relations. (One could also compute directly.) The operators $L_{x^{\prime}}$ and $L_{y^{\prime}}$ map states with $L_{z^{\prime}}=\lambda$ into superpositions of states with $L_{z^{\prime}}=\lambda \pm 1$. Thus, the contribution in the inner product in the fourth term of Eq. (3.5) is zero.

In order for Eq. (3.5) to have a solution for all $x$, we choose $T_{4}(x)$ to be 
multiplication by

$$
T_{4}(x)=\left\langle\Phi(x, \cdot),\left[A+\frac{L^{2}}{2 R_{0}^{2}}+D(0)\right] \Phi(x, \cdot)\right\rangle_{L^{2}\left(\left(x / x^{2}\right) d \alpha d \beta d \gamma\right)} .
$$

With the choice, the fourth and fifth terms on the left-hand side of Eq. (3.5) cancel. We then easily see that $\mathscr{E}_{4}=\left(j(j+1) / 2 R_{0}^{2}\right)+T_{4}\left(R_{0}\right)+($ semiclassical correction terms). This expression is equal to the one given in Remark 5 following Theorem 2.1.

From the components of Eq. (3.4) that are multiples of $\Phi(x, \gamma, \alpha, \beta)$ but orthogonal to $H_{n}(w) e^{-w^{2} / 2}$ we must have

$$
\begin{aligned}
\psi_{2}{ }^{\perp}= & \frac{1}{36}\left[E^{\prime \prime \prime}\left(R_{0}\right)\right]^{2} Y_{j, m}(\theta, \varphi) \Phi(x, \alpha, \beta) \\
& \cdot\left[H_{\mathrm{osc}}-\mathscr{E}_{2}\right]_{r}^{-1} Q_{y} y^{3}\left[H_{\mathrm{osc}}-\mathscr{E}_{2}\right]_{r}^{-1} y^{3} H_{n}(w) e^{-w^{2} / 2} \\
& -\frac{1}{24} E^{\prime \prime \prime \prime}\left(R_{0}\right) Y_{j, m}(\theta, \varphi) \Phi(x, \alpha, \beta)\left[H_{\mathrm{osc}}-\mathscr{E}_{2}\right]^{-1} Q_{y} y^{4} H_{n}(w) e^{-w^{2} / 2},
\end{aligned}
$$

where $Q_{y}$ denotes the orthogonal projection in $L^{2}(d y)$ onto the subspace orthogonal to $H_{n}(w) e^{-w^{2} / 2}$.

The components of Eq. (3.5) that are orthogonal to $\Phi(x, \gamma, \alpha, \beta)$ in $L^{2}\left(\left(\alpha / x^{2}\right) d \alpha d \beta d \gamma\right)$ force us to choose

$$
\begin{aligned}
\psi_{4}^{\perp}= & \frac{1}{6} E^{\prime \prime \prime}\left(R_{0}\right) Y_{j, m}(\theta, \varphi)\left(\frac{\partial}{\partial y}\left[H_{\mathrm{osc}}-\mathscr{E}_{2}\right]_{r}^{-1} y^{3} H_{n}(w) e^{-w^{2} / 2}\right) \\
& \cdot\left([h(x)-E(x)]_{r}^{-1}\left[\frac{\partial \Phi}{\partial x}-\frac{\alpha}{x} \frac{\partial \Phi}{\partial \alpha}-\frac{\beta}{x} \frac{\partial \Phi}{\partial \beta}\right]\right)(x, \alpha, \beta), \\
& -H_{n}(w) e^{-w^{2} / 2}\left([h(x)-E(x)]_{r}^{-1} Q_{r}\right. \\
& \left.\cdot\left(A-\frac{L \cdot J}{R_{0}^{2}}+\frac{L^{2}}{2 R_{0}^{2}}+D(0)\right) Y_{j, m} \Phi\right)(x, \theta, \varphi, \gamma, \alpha, \beta),
\end{aligned}
$$

where $Q_{r}$ denotes the orthogonal projection in $L^{2}\left(\left(\alpha / x^{2}\right) d \alpha d \beta d \gamma\right)$ onto the subspace orthogonal to $\Phi(x, \gamma, \alpha, \beta)$.

From this point on, the $k^{\text {th }}$ order terms Eq. (3.2) recursively yield formulas for $\mathscr{E}_{k}, \psi_{k-2}^{\perp}$, and $\psi_{k}^{\perp}$. The operator $T_{k}$ is chosen so that $g_{k}(x, y)$ is independent of $x$. This choice forces $\psi_{k-4}$ to be a constant multiple of $H_{n}(w) e^{-w^{2} / 2} \Phi(x, \alpha, \beta)$, which we arbitrarily choose to be zero. Explicitly, the $k^{\text {th }}$ order terms require

$$
\begin{aligned}
& {[h(x, \theta, \varphi)-E(x)] \psi_{k}+\left[-\frac{1}{2} \frac{\partial^{2}}{\partial y^{2}}+\frac{1}{2} E^{\prime \prime}\left(R_{0}\right) y^{2}-\mathscr{E}_{2}\right] \psi_{k-2}} \\
& \quad+\sum_{i=3}^{k} \frac{1}{i !} E^{(i)}\left(R_{0}\right) y^{i} \psi_{k-i}-\left[\frac{\partial^{2}}{\partial x \partial y}-\frac{\alpha}{x} \frac{\partial^{2}}{\partial \alpha \partial y}-\frac{\beta}{x} \frac{\partial^{2}}{\partial \beta \partial y}\right] \psi_{k-3} \\
& \quad+A \psi_{k-4}+\sum_{i=0,4, \ldots}^{4[(k-4) / 4]} D^{(i)}(0) \psi_{k-i-4} \\
& \quad+\frac{1}{2}\left[j(j+1)-2 L \cdot J+L^{2}\right] \sum_{i=0}^{k-4}(-1)^{i}(i+1) y^{i} R_{0}^{-i-2} \psi_{k-i-4}
\end{aligned}
$$




$$
\begin{aligned}
& +\sum_{i=4}^{k} \sum_{\ell=0}^{k-i} \frac{1}{\ell !} T_{i}^{(\ell)}\left(R_{0}\right) y^{\ell} \psi_{k-\ell-i}-\sum_{i=4}^{k} T_{i}(x) \psi_{k-i} \\
= & \sum_{i=4}^{k} \mathscr{E}_{i} \psi_{k-i} .
\end{aligned}
$$

The projections of the two sides of Eq. (3.6) into the subspace spanned by $x, \theta$, and $\varphi$ dependent multiples of $H_{n}(w) e^{-w^{2} / 2} \Phi(x, \gamma, \alpha, \beta)$ must be equal. This forces a condition of the form

$$
\begin{gathered}
{\left[G_{k}(x)+T_{k}\left(R_{0}\right)-T_{k}(x)\right] Y_{j, m}(\theta, \varphi) H_{n}(w) e^{-w^{2} / 2} \Phi(x, \gamma, \alpha, \beta)} \\
=\mathscr{E}_{k} Y_{j, m}(\theta, \varphi) H_{n}(w) e^{-w^{2} / 2} \Phi(x, \gamma, \alpha, \beta),
\end{gathered}
$$

where $G_{k}$ is some function of $x$. To solve this, we choose $T_{k}(x)=G_{k}(x)$, and we are forced to have $\mathscr{E}_{k}=T_{k}\left(R_{0}\right)$. Next, the components of the two sides of Eq. (3.6) that are multiples of $\Phi(x, \gamma, \alpha, \beta)$, but orthogonal to $H_{n}(w) e^{-w^{2 / 2}}$ must be equal. This condition takes the form

$$
\left[H_{\text {osc }}-\mathscr{E}_{2}\right] \psi_{k-2}^{\perp}=(\text { a determinable function }),
$$

and determines $\psi_{h-2}^{\perp}$. Finally, the components of Eq. (3.6) that are orthogonal to $\Phi(x, \gamma, \alpha, \beta)$ require a condition of the form

$$
[h(x, \theta, \varphi)-E(x)] \psi_{k}^{\perp}=(\text { a determinable function }) .
$$

This determines $\psi_{k}^{\perp}$.

In the particular case of $k=5$, one finds that the function $G_{5}(x)=0$ because of symmetries in the calculation. Thus, we take $T_{5}(x)=0$ and have $\mathscr{E}_{5}=0$. The coupling of electronic and nuclear motions destroys these symmetries in higher orders.

The case of $\Lambda \neq 0$ is not substantially different, except that one must use degenerate perturbation theory on the pairs of states with $L_{z^{\prime}}= \pm \Lambda$. The only term that couples these two states is the term containing $L \cdot J$. The angular momentum selection rules to which we referred in the discussion of the fourth order terms prevent any coupling of the $\Lambda$ and $-\Lambda$ states before order $\varepsilon^{8|\Lambda|}$. Before one reaches that order, there is no complication, except that one must always handle two states instead of one, and the energy will contain an occasional extra term (e.g., at fourth order, there is an additional term due to the fact that $L_{z^{\prime}} \cdot J_{z^{\prime}}$ is no longer zero, but $\Lambda^{2}$ ). Although we have not explicitly done the perturbation calculations through order $\varepsilon^{8, \Lambda \mid}$ in any examples, we believe that standard physicists formal perturbation scheme can be applied without any difficulty. We except the degeneracy to be broken at order $\varepsilon^{8|\Lambda|}$.

3B. More Than One Electron. The case of more than one electron leads to more messy formulas, but does not require any substantially new ideas. So, we will not go through the perturbation calculations in detail, but simply do the beginning of the multiple scales argument, and describe how to proceed.

In the original independent variables, $\left(R, \theta, \varphi, \zeta_{2}, \ldots, \zeta_{N-1}\right)$, the Hamiltonian 
has the form

$$
H(\varepsilon)=-\frac{\varepsilon^{4}}{2}\left(\frac{\partial^{2}}{\partial R^{2}}-\frac{N^{2}}{R^{2}}\right)+h(R, \theta, \varphi),
$$

where

$$
N^{2}=-\frac{\partial^{2}}{\partial \theta^{2}}-\cot \theta \frac{\partial}{\partial \theta}-\frac{1}{\sin ^{2} \theta} \frac{\partial^{2}}{\partial \varphi^{2}} .
$$

To obtain the Hamiltonian in the new variables, we proceed in two steps. First, we change to the rotating coordinates for the electrons. Then, we dilate the electronic coordinates. When doing the first step, the only terms in the Hamiltonian that are altered are the ones that appear in $N^{2}$ and in the potential energy. The alteration of the potential energy is straightforward. The alteration of $N^{2}$ is done by replacing $\partial / \partial \theta$ and $\partial / \partial \varphi$ by new expressions. The old $\partial / \partial \theta$ is replaced by

$$
\begin{gathered}
\frac{\partial}{\partial \theta}+\cos \gamma\left(\rho \frac{\partial}{\partial z^{\prime}}-z^{\prime} \frac{\partial}{\partial \rho}\right)+\frac{z^{\prime}}{\rho} \sin \gamma \frac{\partial}{\partial \gamma} \\
+\sum_{j=2}^{N-2}\left(\eta_{j}^{(1)} \frac{\partial}{\partial \eta_{j}^{(3)}}-\eta_{j}^{(3)} \frac{\partial}{\partial \eta_{j}^{(1)}}\right) .
\end{gathered}
$$

The old $\partial / \partial \varphi$ is replaced by

$$
\begin{aligned}
\frac{\partial}{\partial \varphi} & +\sin \theta\left[\sin \gamma\left(\rho \frac{\partial}{\partial z^{\prime}}-z^{\prime} \frac{\partial}{\partial \rho}\right)-\frac{z^{\prime}}{\rho} \cos \gamma \frac{\partial}{\partial \gamma}\right. \\
& \left.+\sum_{j=2}^{N-2}\left(\eta_{j}^{(2)} \frac{\partial}{\partial \eta_{j}^{(3)}}-\eta_{j}^{(3)} \frac{\partial}{\partial \eta_{j}^{(2)}}\right)\right] \\
& -\cos \theta\left[\frac{\partial}{\partial \gamma}+\sum_{j=2}^{N-2}\left(\eta_{j}^{(1)} \frac{\partial}{\partial \eta_{j}^{(2)}}-\eta_{j}^{(2)} \frac{\partial}{\partial \eta_{j}^{(1)}}\right)\right] .
\end{aligned}
$$

After these replacements are made, the product rule for computing derivatives produces a complicated formula for $N^{2}$, but it still has the form $J^{2}-2 L \cdot J+L^{2}$, where $J^{2}$ is as in the one electron case.

The second step in changing coordinates is accomplished by dilating the variables $\rho, z^{\prime}, \eta_{2}, \ldots, \eta_{N-2}$ by $R$ to obtain $\alpha, \beta, \tau_{2}, \ldots, \tau_{N-2}$, as described in Sect. 2. The only complications in the expression for $H(\varepsilon)$ are that

$$
\frac{\partial}{\partial \rho}=\frac{1}{R} \frac{\partial}{\partial \alpha}, \quad \frac{\partial}{\partial z}=\frac{1}{R} \frac{\partial}{\partial \beta}, \quad \nabla_{\xi_{j}}=\frac{1}{R} \nabla_{\tau_{j}}
$$

and that $\partial / \partial R$ is replaced by

$$
\frac{\partial}{\partial R}-\frac{\alpha}{R} \frac{\partial}{\partial \alpha}-\frac{\beta}{R} \frac{\partial}{\partial \beta}-\frac{1}{R} \sum_{j=2}^{N-2} \tau_{j} \cdot \nabla_{\tau_{j}} .
$$

After these calculations are completed, one does the multiple scales expansion exactly as in Sect. 3A. 


\section{Proof of the Main Theorems}

We now must show that the formal expressions of Sect. 3 define rigorous quasimodes. The main difficulty in this task is proving existence of all the quantities defined formally in Sect. 3.

We let $r=\left(\zeta_{1}, \zeta_{2}, \ldots, \zeta_{N-2}\right)$ be the original electronic clustered Jacobi coordinates. We define the weighted Sobolev spaces

$$
\mathscr{H}_{1, s}=\left\{f \in \mathscr{D}^{\prime}\left(\mathbb{R}^{3 N-6}\right):\left(1+r^{2}\right)^{s / 2}(1-\Delta)^{\prime / 2} f \in L^{2}\left(\mathbb{R}^{3 N-6}\right)\right\} .
$$

We let $\mathscr{K}_{t, s}$ denote the corresponding space in the variables $\left(\gamma, \alpha, \beta, \tau_{2}, \ldots, \tau_{N-2}\right)$ of Sect. 2, i.e., $\mathscr{K}_{t, s}=\left\{g: g\left(\gamma, \alpha, \beta, \tau_{2}, \ldots, \tau_{N-2}\right)=f\left(\zeta_{1}, \zeta_{2}, \ldots, \zeta_{N-2}\right)\right.$ with $\left.f \in \mathscr{H}_{t, s}\right\}$.

Lemma 4.1. Let $h(R, \theta, \varphi)$ denote the electron Hamiltonian in the coordinates $\left(R, \theta, \varphi, \gamma, \alpha, \beta, \tau_{2}, \ldots, \tau_{N-2}\right)$. Let $E(R)$ be a discrete eigenvalue of $h(R, \theta, \varphi)$ for $R \in U$, with associated spectral projection $P(R, \theta, \varphi)$. Let $n(R, \theta, \varphi, z)=(z-h(R, \theta, \varphi))^{-1}$, and let $\tilde{n}(R, \theta, \varphi, z)=n(R, \theta, \varphi, z)(1-P(R, \theta, \varphi))$. Then $\tilde{n}(R, \theta, \varphi, E(R))$ is a bounded analytic family from $\mathscr{K}_{0, s}$ to $\mathscr{K}_{2, s}$ for all $s$.

Proof. For any operator $A$ in the coordinates $\left(\gamma, \alpha, \beta, \tau_{2}, \ldots, \tau_{N-2}\right)$, we let $A^{\#}$ denote the corresponding operator in the coordinates $\left(\zeta_{1}, \zeta_{2}, \ldots, \zeta_{N-2}\right)$. It is sufficient to do all calculations in the $\zeta$ coordinates, and prove boundedness of $\tilde{n}^{\#}(R, \theta, \varphi, E(R))$ from $\mathscr{H}_{0 . s}$ to $\mathscr{H}_{2 . s}$. We first prove that the resolvement $\tilde{n}_{0}(z)$ of the free electron Hamiltonian is bounded from $\mathscr{K}_{0, s}$ to $\mathscr{K}_{2, s}$ for all $s$ if $z \notin[0, \infty)$. This is equivalent to showing that the operator

$$
A^{\#}(z)=\left(1+r^{2}\right)^{s / 2}(1-\Delta)(z+\Delta)^{-1}\left(1+r^{2}\right)^{-s / 2}
$$

is bounded on $L^{2}$. To prove this, we rewrite $A^{\#}(z)$ as

$$
\begin{aligned}
A^{\#}(z)= & \left(1+r^{2}\right)^{s / 2}(1-\Delta)(-1+\Delta)^{-1}\left(1+r^{2}\right)^{-s / 2} \\
& -(1+z)\left(1+r^{2}\right)^{s / 2}(1-\Delta)(-1+\Delta)^{-1}\left(1+r^{2}\right)^{-s / 2} \\
& \cdot\left(1+r^{2}\right)^{s / 2}(z+\Delta)^{-1}\left(1+r^{2}\right)^{-s / 2} \\
= & -1+(1+z)\left(1+r^{2}\right)^{s / 2}(z+\Delta)^{-1}\left(1+r^{2}\right)^{-s / 2} .
\end{aligned}
$$

This last expression is bounded on $L^{2}$ by Lemma 1 on page 170 of [20].

Next, we show that if $(R, \theta, \varphi)$ is fixed and $z$ is not in the spectrum of $h^{\#}(R, \theta, \varphi)$, then $n^{\#}(R, \theta, \varphi, z)$ is bounded from $\mathscr{H}_{0, s}$ to $\mathscr{H}_{2, s}$ for all $s$. This is equivalent to proving

$$
B^{\#}(z)=\left(1+r^{2}\right)^{s / 2}(1-\Delta) n^{\#}(R, \theta, \varphi, z)\left(1+r^{2}\right)^{-s / 2}
$$

is bounded on $L^{2}$. To prove this, we rewrite $B^{\#}(z)$ as

$$
\begin{aligned}
B^{\#}(z)= & \left(1+r^{2}\right)^{s / 2}(1-\Delta) n_{0}^{\#}(R, \theta, \varphi, z)\left(1+r^{2}\right)^{-s / 2} \\
& \cdot\left(1+r^{2}\right)^{s / 2}\left[1-V n_{0}^{\#}(R, \theta, \varphi, z)\right]^{-1}\left(1+r^{2}\right)^{-s / 2} \\
= & A^{\#}(z)\left(1+r^{2}\right)^{s / 2}\left[1-V n_{0}^{\#}(R, \theta, \varphi, z)\right]^{-1}\left(1+r^{2}\right)^{-s / 2} .
\end{aligned}
$$

Since $A^{\#}(z)$ is bounded, we need only show

$$
\left(1+r^{2}\right)^{s / 2}\left[1-V n_{0}^{\#}(R, \theta, \varphi, z)\right]^{-1}\left(1+r^{2}\right)^{-s / 2}
$$


is bounded on $L^{2}$. This follows from a simple many-body extension of the proof of Lemma 1 on page 170 of [20].

The operator $P^{\#}(R, \theta, \varphi)$ maps $\mathscr{H}_{0, s}$ into $\mathscr{H}_{0, s}$ because of the exponential fall of discrete eigenfunctions $[1,2,9-11,19,20]$. Thus, by the above, $n^{\#}(R, \theta, \varphi, z)$ $\left(1-P^{\#}(R, \theta, \varphi)\right)$ is bounded from $\mathscr{H}_{0, s}$ into $\mathscr{H}_{2, s}$ for all $z \notin \sigma\left(h^{\#}(R, \theta, \varphi)\right)$ and all $s$. This bound is uniform for $z$ in compact subsets of the complement of $\sigma(h(R, \theta, \varphi))$. The boundedness part of the lemma follows from this by use of the Cauchy integral

$$
\tilde{n}^{\#}(R, \theta, \varphi, E(R))=\frac{1}{2 \pi i} \int_{\Gamma} \frac{d z}{E-z} n^{\#}(R, \theta, \varphi, z)\left(1-P^{\#}(R, \theta, \varphi)\right),
$$

where $\Gamma$ is a small circle around the point $E(R)$. The proof of analyticity is similar.

Definition. Let $\mathscr{A}_{\ell, s}^{(j, m)}$ be the set of all $C^{\infty}$ functions $f(x, y, \theta, \varphi)(x \in U)$ with values in $\mathscr{K}_{\ell, s}$, such that $J^{2} f=j(j+1) f$ and $J_{z} f=m f$. Let $\mathscr{A}_{\ell}^{(j, m)}=\bigcap_{s} \mathscr{A}_{p, s}^{(j, m)}$.

Lemma 4.2. Let $\mathscr{M}$ be any operator that occurs in Eq. (3.6) other than $[h(x, \theta, \varphi)-$ $E(x)], T_{i}(x)$, or $T_{i}^{(\ell)}\left(R_{0}\right)$ (in the multi-electron case, Eq. (3.6) must be replaced by its multi-electron analog). Then $\mathscr{M}$ maps $\mathscr{A}_{2}^{(j, m)}$ into $\mathscr{A}_{0}^{(j, m)}$.

Proof. First we note that the operators of Eq. (3.6) (and its multi-electron analogs) commute with $J^{2}$ and $J_{z}$. Furthermore, functions in $\mathscr{A}_{f}^{(j, m)}$ have the form

$$
f\left(x, y, \theta, \varphi, \gamma, \alpha, \beta, \tau_{2}, \ldots, \tau_{N-2}\right)=\sum_{\lambda=-j}^{j} \mathscr{D}_{m, \lambda}^{(j)}(\theta, \varphi, \gamma) f_{\lambda}\left(x, y, \alpha, \beta, \tau_{2}, \ldots, \tau_{N-2}\right),
$$

where the $\mathscr{D}_{m, \hat{\lambda}}^{(j)}(\theta, \varphi, \gamma)$ are $C^{\infty}[11]$. Thus, the smoothness in $\theta$ and $\varphi($ and $\gamma)$ is clear.

Second, as in the proof of Lemma 4.1, it is sufficient to work in the original electronic coordinate system and prove the analogous result in that representation. In those coordinates, $\mathscr{M}$ is an at most second order differential operator with $C^{\infty}$ coefficients that grow at most polynomially.

The lemma follows from these observations.

Lemma 4.3. The formal expressions for the $\psi_{k}$ 's in Sect. 3 define functions that belong to $\mathscr{A}_{2}^{(j, m)}$.

Proof. The electron wave function $\Phi(x)$ is analytic in $x \in U$, and is independent of $\theta$, and $\varphi$. It belongs to $\mathscr{H}_{2, s}$ for all $s$ because of exponential fall off $[1,2,9-11,19,20]$, the fact that $h(x) \Phi(x)=E(x) \Phi(x)$, and the fact that $h(x, \theta, \varphi)$ has the same domain as the electronic kinetic energy. Thus, the explicit formulas for $\psi_{0}, \psi_{1}=$ $\psi_{1}^{\perp}$ and $\psi_{2}=\psi_{2}^{\perp}$ show that these functions exist in $\mathscr{A}_{2}^{(j, m)}$. Similarly, the explicit formulas and lemmas. 4.1 and 4.2 show that $\psi_{3}^{\perp}$ and $\psi_{4}^{\perp}$ belong to $\mathscr{A}_{2}^{(j, m)}$, and that $T_{4}(x)$ is a $C^{\infty}$ function of $x$.

We now proceed by induction on $k \geqq 5$. Assume $\mathscr{E}_{0}, \mathscr{E}_{1}, \ldots, \mathscr{E}_{k-1}$ are determined; $\psi_{0}, \psi_{1}, \ldots, \psi_{k-3}, \psi_{k-2}^{\perp}$, and $\psi_{k-1}^{\perp}$ belong to $\mathscr{A}_{2}^{(j, m)}$; and that $T_{4}(x), T_{5}(x), \ldots, T_{k-1}(x)$ belong to $C^{\infty}(U)$.

By Lemma 4.2 and the discussion following Eq. (3.6), $T_{k}(x)$ is $C^{\infty}$ on $U$ and $\mathscr{E}_{k}$ is determined. Similarly, $\psi_{k-2}^{\perp}$ is determined in $\mathscr{A}_{2}^{(j, m)}$. Finally, Lemmas 4.1 and 
4.2 and the discussion following Eq. (3.6) show that $\psi_{n}^{\perp}$ exists in $\mathscr{A}_{2}^{(j, m)}$. Thus, the induction can proceed.

Proof of the Theorems. Choose $K \geqq 4$; let

$$
\begin{aligned}
\psi_{\varepsilon} & =\sum_{n=0}^{K-4} \varepsilon^{n} \psi_{n}+\sum_{n=K-4}^{K-2} \varepsilon^{n} \psi_{n}^{\perp}+\sum_{n=K-4}^{K} \varepsilon^{n} \psi_{n}^{\perp}, \\
\mathscr{E}(\varepsilon) & =\sum_{n=0}^{K} \varepsilon^{n} \mathscr{E}_{n}, \\
\Psi_{\varepsilon}(R, \theta, \varphi, r) & =R^{-1} \psi_{\varepsilon}\left(R, \frac{R-R_{0}}{\varepsilon}, \theta, \varphi, r\right) F(R) ;
\end{aligned}
$$

and compute

$$
\{H(\varepsilon)-\mathscr{E}(\varepsilon)\} \Psi_{\varepsilon}(R, \theta, \varphi, r) .
$$

Because the $\psi_{n}$ 's satisfy the formal equations of Sect. 3, all terms in this expression cancel, except for two types. The first type involves derivatives of $F$. The $R$ dependence of these terms involves products of bounded functions of $R$ with support away from $R_{0}$, times polynomials in $\left[E^{\prime \prime}\left(R_{0}\right)\right]^{1 / 2}\left(R-R_{0}\right) / \varepsilon$, times $e^{-E^{\prime \prime}\left(R_{0}\right)\left(R-R_{0}\right)^{2} / 2 \varepsilon^{2}}$. Such functions are easily seen to have norms that are smaller than $\varepsilon^{n}$ for any $n$. The second type of term has the form of a function whose norm is bounded as $\varepsilon$ tends to zero, times $\varepsilon^{n}$, for some $n \geqq K$. Thus, by the triangle inequality, the norm of

$$
\{H(\varepsilon)-\mathscr{E}(\varepsilon)\} \Psi_{\varepsilon}(R, \theta, \varphi, r)
$$

is bounded by a multiple of $\varepsilon^{K+1}$, and $\Psi_{\varepsilon}$ is a quasimode of order $K$. The theorems of Sect. 2 follow from this.

\section{References}

1. Agmon, S.: Lectures on exponential decay of solutions of second-order elliptic equations: Bounds on eigenfunctions of $N$-body Schrödinger operators. Princeton, New Jersey: Princeton University Press 1982

2. Agmon, S.: Bounds on exponential decay of eigenfunctions of Schrödinger operators. In: Schrödinger Operators. Graffi, S. (ed.) Lecture Notes in Mathematics Vol. 1159, pp.1-38. Berlin, Heidelberg, New York, Tokyo: Springer 1985

3. Aventini, P., Seiler, R.: On the electronic spectrum of the diatomic molecular ion. Commun. Math. Phys. 41, 119-134 (1975)

4. Born, M., Oppenheimer, R.: Zur Quantentheorie der Molekeln. Ann. Phys. (Leipzig) 84, 457-484 (1927)

5. Combes, J.-M.: On the Born-Oppenheimer approximation. In: International Symposium on Mathematical Problems in Theoretical Physics. Araki, H. (ed.), pp. 467-471. Berlin, Heidelberg, New York: Springer 1975

6. Combes, J.-M.: The Born-Oppenheimer approximation. In: The Schrödinger equation. Thirring, W., Urban, P. (eds.), pp. 139-159. Wien, New York: Springei 1977

7. Combes, J.-M., Duclos, P., Seiler, R.: The Born-Oppenheimer approximation. In: Rigorous atomic and molecular physics. Velo, G., Wightman, A. (eds.), pp. 185-212. New York: Plenum 1981

8. Combes, J.-M., Seiler, R.: Regularity and asymptotic properties of the discrete spectrum of electronic Hamiltonians. Int. J. Quantum Chem. 14, 213-229 (1978)

9. Combes, J.-M., Thomas, L.: Asymptotic behavior of eigenfunctions for multiparticle Schrödinger operators. Commun. Math. Phys. 34, 251-270 (1973) 
10. Deift, P., Hunziker, W., Simon, B., Vock, E.: Pointwise bounds on eigenfunctions and wave packets in $N$-body quantum systems IV. Commun. Math. Phys. 64, 1-34 (1978)

11. Edmonds, A. R.: Angular momentum in quantum mechanics (2nd ed.). Princeton, New Jersey: Princeton University Press 1960

12. Froese, R., Herbst, I.: Exponential bounds and absence of positive eigenvalues for $N$-body Schrödinger operators. Commun. Math. Phys. 87, 429-447 (1983)

13. Hagedorn, G. A.: A time dependent Born-Oppenheimer approximation. Commun. Math. Phys. 77, $1-19(1980)$

14. Hagedorn, G. A.: High order corrections to the tıme-dependent Born-Oppenheimer approximation I: Smooth potentials. Ann. Math. 124, $571-590$ (1986)

15. Hagedorn, G. A.: High order corrections to the time-independent Born-Oppenheimer approximation I: Smooth potentials. Ann. Inst. H. Poincaré Sect. A. 47, 1-16 (1987)

16. Hunziker, W., Sigal, I. M.: Distortion analyticity and molecular resonance curves. E.T.H. preprint, Oct. 1985

17. Judd, B. R.: Angular momentum theory for diatomic molecules. New York, San Francisco, London: Academic Press 1975

18. Landau, L. D., Lifshitz, E. M.: Quantum mechanics (Non-relativistic Theory) (3rd revised ed.). Oxford, New York, Toronto, Sydney, Pairs, Frankfurt: Pergamon Press 1977

19. O'Connor, A. J.: Exponential decay of bound state wave functions. Commun. Math. Phys. 32, 319-340 (1973)

20. Reed, M., Simon, B.: Methods of modern mathematical physics Vol. IV analysis of operators. New York, London: Academic Press 1978

21. Seiler, R.: Does the Born-Oppenheimer approximation work? Helv. Phys. Acta 46, 230-234 (1973)

22. Simon, B.: Semiclassical analysis of low lying eigenvalues. I. Non-degenerate minima: Asymptotic expansions. Ann. Inst. H. Poincaré Sect. A. 38, 295-308 (1983)

23. Udim, T.: Schrödinger operatoren mit stark singularen spin-wechselwirkung. Arch. Math. 38, $357-365$ (1982)

Communicated by B. Simon

Received January 9, 1987; in revised form October 6, 1987 